OPEN ACCESS

Edited and reviewed by: Sairah Ahmed,

University of Texas MD Anderson

Cancer Center, United States

*Correspondence:

JianChao Huang

literatusyanyun@163.com

${ }^{\dagger}$ These authors have contributed equally to this work and share first authorship

Specialty section: This article was submitted to

Cancer Immunity and Immunotherapy, a section of the journal

Frontiers in Oncology

Received: 31 August 2021 Accepted: 08 September 2021 Published: 07 October 2021

Citation:

Meng J, Wu X, Sun Z, Xun R, Liu M,

Hu R and Huang J (2021) Corrigendum: Efficacy and Safety of CAR-T Cell Products Axicabtagene Ciloleucel, Tisagenlecleucel, and Lisocabtagene Maraleucel for the

Treatment of Hematologic

Malignancies: A Systematic

Review and Meta-Analysis.

Front. Oncol. 11:768128.

doi: 10.3389/fonc.2021.768128

\section{Corrigendum: Efficacy and Safety of CAR-T Cell Products Axicabtagene Ciloleucel, Tisagenlecleucel, and Lisocabtagene Maraleucel for the Treatment of Hematologic Malignancies: A Systematic Review and Meta-Analysis}

\author{
Jun Meng ${ }^{1+}$, XiaoQin $\mathrm{Wu}^{2 \dagger}$, Zhen Sun ${ }^{3}$, RenDe Xun ${ }^{2}$, MengSi $\mathrm{Liu}^{3}$, Rui Hu${ }^{3}$ \\ and JianChao Huang ${ }^{2 *}$ \\ ${ }^{1}$ Molecular Genetics Laboratory, Suining Central Hospital, Suining, China, 2 Department of Neurosurgery, The First Affiliated \\ Hospital, University of South China, Hengyang, China, ${ }^{3}$ Hengyang Medical College, University of South China, Hengyang, China
}

Keywords: chimeric antigen receptor T-cell product, CAR-T cell therapy, immunotherapy, lymphoma, leukemia, hematologic malignancy, efficacy, safety

\section{A Corrigendum on:}

Efficacy and Safety of CAR-T Cell Products Axicabtagene Ciloleucel, Tisagenlecleucel, and Lisocabtagene Maraleucel for the Treatment of Hematologic Malignancies: A Systematic Review and Meta-Analysis

By Meng J, Wu X, Sun Z, Xun R, Liu M, Hu R and Huang J (2021). Front. Oncol. 11:698607. doi: 10.3389/fonc.2021.698607

In the original article, there were mistakes in Table $\mathbf{2}$ as published. We collected data with reference to both the conference abstract version and the full-text published version of the TRANSCEND NHL 001 trial conducted by Abramson et al. $(1,2)$; in Table 2, we showed the data from the conference abstract version, which should be changed to the full-text version. The rest of the paper, 
TABLE 2 | Characteristics of included studies.

\begin{tabular}{|c|c|c|c|c|c|c|c|c|c|c|}
\hline $\begin{array}{l}\text { First } \\
\text { Author }\end{array}$ & Year & No. & $\begin{array}{l}\text { Median age } \\
\text { (range)-yr }\end{array}$ & Histological type & CAR-T type & $\begin{array}{c}\text { Efficacy } \\
\text { evaluation }\end{array}$ & Scale & $\begin{array}{l}\text { Toxicity evaluation } \\
\text { (grade } \geq 3 \text { ) }\end{array}$ & Scale (CRS/ICANS) & Ref \\
\hline Schuster & 2018 & 111 & $56(22-76)$ & DLBCL & tisa-cel & $\begin{array}{l}\text { CR: } 37 / 93 \\
\text { PR: } 11 / 93\end{array}$ & Lugano & $\begin{array}{l}\text { CRS: } 24 / 111 \\
\text { ICANS: } 13 / 111\end{array}$ & $\begin{array}{l}\text { Penn/CTCAE 4.03, } \\
\text { MDRA } 20.1\end{array}$ & 17 \\
\hline Schubert & 2020 & 21 & $52(20-68)$ & $\begin{array}{l}16 \text { DLBCL, } 3 \text { PMBCL, } 1 \\
\text { DHL, } 1 \mathrm{tFL}\end{array}$ & axi-cel & $\begin{array}{l}\text { CR: 9/21 } \\
\text { PR: 10/21 }\end{array}$ & Lugano & $\begin{array}{l}\text { CRS: } 0 / 21 \\
\text { ICANS: } 6 / 21\end{array}$ & ASTCT/ASTCT & 22 \\
\hline Pinnix & 2020 & 124 & $60(18-85)$ & $\begin{array}{l}95 \text { DLBCL, } 20 \text { tFL, } 9 \\
\text { PMBCL }\end{array}$ & axi-cel & $\begin{array}{l}\text { CR: 60/ } \\
124 \\
\text { PR: 36/ } \\
124\end{array}$ & Lugano & $\begin{array}{l}\text { CRS: } 11 / 124 \\
\text { ICANS: } 49 / 124\end{array}$ & $\begin{array}{l}\text { ASTCT, CARTOX } \\
\text { ASTCT, CARTOX }\end{array}$ & 23 \\
\hline Nastoupil & 2020 & 298 & $60(21-83)$ & $\begin{array}{l}203 \text { DLBCL, } 76 \text { tFL, } 19 \\
\text { PMBCL }\end{array}$ & axi-cel & $\begin{array}{l}\text { CR: 175/ } \\
275 \\
\text { PR: 50/ } \\
175\end{array}$ & Lugano & $\begin{array}{l}\text { CRS: } 19 / 275 \\
\text { ICANS: } 85 / 275\end{array}$ & $\begin{array}{l}\text { CARTOX, Lee/ } \\
\text { CARTOX, CTCAE } \\
4.03\end{array}$ & 24 \\
\hline Neelapu & 2017 & 101 & $58(23-76)$ & $\begin{array}{l}77 \text { DLBCL, } 16 \text { tFL, } 8 \\
\text { PMBCL }\end{array}$ & axi-cel & $\begin{array}{l}\text { CR: 55/ } \\
101 \\
\text { PR: 28/ } \\
101\end{array}$ & IWGRC & $\begin{array}{l}\text { CRS: } 13 / 101 \\
\text { ICANS: } 28 / 101\end{array}$ & Lee/CTCAE 4.03 & 19 \\
\hline Locke & 2017 & 7 & $46(29-69)$ & DLBCL & axi-cel & $\begin{array}{l}\text { CR: } 4 / 7 \\
\text { PR: } 1 / 7\end{array}$ & IWGRC & $\begin{array}{l}\text { CRS: } 1 / 7 \\
\text { ICANS: } 4 / 7\end{array}$ & Lee/CTCAE 4.03 & 25 \\
\hline Jain & 2019 & 4 & $56(38-66)$ & DLBCL & axi-cel & $\begin{array}{l}\text { CR: } 2 / 4 \\
\text { PR: } 1 / 4\end{array}$ & NP & $\begin{array}{l}\text { CRS: } 0 / 4 \\
\text { ICANS: } 0 / 4\end{array}$ & NP/NP & 26 \\
\hline Abbasi & 2020 & 10 & $66(55-77)$ & DLBCL & axi-cel & $\begin{array}{l}\text { CR: } 8 / 10 \\
\text { PR: } 0 / 10\end{array}$ & NP & $\begin{array}{l}\text { CRS: } 1 / 10 \\
\text { ICANS: } 3 / 10\end{array}$ & ASTCT/ASTCT & 27 \\
\hline Garfall & 2018 & 10 & $61(48-68)$ & MM & tisa-cel & $\begin{array}{l}\text { CR: } 6 / 10^{\dagger} \\
\text { PR: } 2 / 10\end{array}$ & IMWGRC & $\begin{array}{l}\text { CRS: } 0 / 10 \\
\text { ICANS: } 0 / 10\end{array}$ & NP/NP & 28 \\
\hline Maude & 2018 & 75 & $11(3-23)$ & ALL & tisa-cel & $\begin{array}{l}\text { CR: } 61 / 75 \\
\text { PR: } 0 / 75\end{array}$ & $\begin{array}{l}\text { Independent } \\
\text { scale }\end{array}$ & $\begin{array}{l}\text { CRS: } 35 / 75 \\
\text { ICANS: } 10 / 75\end{array}$ & Penn/CTCAE 4.03 & 29 \\
\hline Maude & 2014 & 30 & $14(5-60)$ & ALL & tisa-cel & $\begin{array}{l}\text { CR: } 27 / 30 \\
\text { PR: } 0 / 30\end{array}$ & $\begin{array}{l}\text { Independent } \\
\text { scale }\end{array}$ & $\begin{array}{l}\text { CRS: } 8 / 30^{\circ} \\
\text { ICANS: NP }\end{array}$ & $\begin{array}{l}\text { Independent scale/ } \\
\text { NP }\end{array}$ & 30 \\
\hline Schuster & 2017 & 28 & $58(25-77)$ & $\begin{array}{l}14 \mathrm{DLBCL} \\
14 \mathrm{FL}\end{array}$ & tisa-cel & $\begin{array}{l}\text { CR: } 16 / 28 \\
\text { PR: } 2 / 28\end{array}$ & $\begin{array}{l}1999 \\
\text { IWGRC }\end{array}$ & $\begin{array}{l}\text { CRS: } 5 / 28 \\
\text { ICANS: } 3 / 28\end{array}$ & Penn/NP & 31 \\
\hline Frigault & 2019 & 8 & $50(17-79)$ & $\begin{array}{l}5 \text { DLBCL, } 2 \text { HGBCL, } 1 \\
\text { PMBCL }\end{array}$ & tisa-cel & $\begin{array}{l}\text { CR: } 2 / 8 \\
\text { PR: } 2 / 8\end{array}$ & NP & $\begin{array}{l}\text { CRS: } 0 / 8 \\
\text { ICANS: } 0 / 8\end{array}$ & $\begin{array}{l}\text { Lee, ASTCT/Lee, } \\
\text { ASTCT }\end{array}$ & 32 \\
\hline Sim & 2019 & 11 & NP & 8 DLBCL, 3 tFL, & axi-cel & $\begin{array}{l}\text { CR: } 5 / 11 \\
\text { PR: } 4 / 11\end{array}$ & Lugano & $\begin{array}{l}\text { CRS: } 1 / 11 \\
\text { ICANS: } 3 / 11\end{array}$ & $\begin{array}{l}\text { CTCAE 5.0/CTCAE } \\
5.0\end{array}$ & 33 \\
\hline Porter & 2015 & 14 & $66(51-78)$ & CLL & tisa-cel & $\begin{array}{l}\text { CR: } 4 / 14 \\
\text { PR: } 4 / 14\end{array}$ & $\begin{array}{l}\text { IWG on CLL } \\
\text { RC }\end{array}$ & $\begin{array}{l}\text { CRS: } 7 / 14 \\
\text { ICANS: } 1 / 14\end{array}$ & Penn/CTCAE 3.0 & 34 \\
\hline Shah & 2018 & 7 & NP & 3 DLBCL, 4 FL & tisa-cel & $\begin{array}{l}\text { CR: } 3 / 7 \\
\text { PR: } 2 / 7\end{array}$ & Lugano & $\begin{array}{l}\text { CRS: NP } \\
\text { ICANS: NP }\end{array}$ & NP/NP & 35 \\
\hline Wright & 2020 & 31 & NP & $26 \mathrm{DLBCL}, 5 \mathrm{tFL}$ & $\begin{array}{l}18 \text { axi-cel, } 13 \\
\text { tisa-cel }\end{array}$ & $\begin{array}{l}\text { CR: } 11 / 27 \\
\text { PR: } 3 / 27\end{array}$ & Lugano & $\begin{array}{l}\text { CRS: } 6 / 31 \\
\text { ICANS: } 4 / 31\end{array}$ & Penn/NP & 36 \\
\hline Jacobson & 2020 & 122 & $62(21-79)$ & $\begin{array}{l}57 \text { DLBCL, } 33 \text { tFL, } 17 \\
\text { HGBCL, } 8 \text { PMBCL, } 5 \text { TMZL, } \\
2 \text { RS }\end{array}$ & axi-cel & $\begin{array}{l}\text { CR: } 61 / 122 \\
\text { PR: 24/122 }\end{array}$ & Lugano & $\begin{array}{l}\text { CRS: } 19 / 122 \\
\text { ICANS: } 43 / 122\end{array}$ & Lee/CTCAE 4.03 & 37 \\
\hline Abramson & 2020 & 269 & $63(54-70)$ & $\begin{array}{l}215 \text { DLBCL, } 36 \text { HGBCL, } 15 \\
\text { PMBCL, } 3 \text { FL3B }\end{array}$ & liso-cel & $\begin{array}{l}\text { CR: 136/ } \\
256 \\
\text { PR: 50/ } \\
256\end{array}$ & Lugano & $\begin{array}{l}\text { CRS: } 6 / 269 \\
\text { ICANS: } 27 / 269\end{array}$ & Lee/CTCAE 4.03 & 16 \\
\hline Fehse & 2019 & 10 & $56(24-79)$ & 7 DLBCL, 3 PMBCL & axi-cel & $\begin{array}{l}\text { CR: } 2 / 10 \\
\text { PR: } 5 / 10\end{array}$ & NP & $\begin{array}{l}\text { CRS: } 2 / 10 \\
\text { ICANS: } 1 / 10\end{array}$ & ASTCT/ASTCT & 38 \\
\hline Gupta & 2019 & 78 & $60+-13 ※$ & DLBCL & $\begin{array}{l}69 \text { axi-cel, } 9 \\
\text { tisa-cel }\end{array}$ & $\begin{array}{l}\text { CR+PR: } \\
43 / 78^{*}\end{array}$ & NP & $\begin{array}{l}\text { CRS: } 10 / 78 \\
\text { ICANS: } 22 / 78\end{array}$ & $\begin{array}{l}\text { CTCAE 5.0, Lee/ } \\
\text { CTCAE } 5.0\end{array}$ & 39 \\
\hline Korell & 2020 & 25 & $54(20-68)$ & 24 DLBCL, 1 PMBCL & axi-cel & $\begin{array}{l}\text { CR: } 9 / 25 \\
\text { PR: } 10 / 25\end{array}$ & Lugano & $\begin{array}{l}\text { CRS: NP } \\
\text { ICANS: NP }\end{array}$ & NP/NP & 40 \\
\hline Frey & 2019 & 35 & $34(21-70)$ & ALL & tisa-cel & $\begin{array}{l}\text { CR: } 24 / 35 \\
\text { PR: } 0 / 35\end{array}$ & $\begin{array}{l}\text { Independent } \\
\text { scale }\end{array}$ & $\begin{array}{l}\text { CRS: } 25 / 35 \\
\text { ICANS: } 2 / 35\end{array}$ & Penn/CTCAE 4.03 & 41 \\
\hline Sesques & 2020 & 61 & $59(27-75)$ & $\begin{array}{l}38 \text { DLBCL, } 18 \text { PMBCL, } 4 \\
\text { tFL, } 1 \text { TMZL }\end{array}$ & $\begin{array}{l}28 \text { axi-cel, } 33 \\
\text { tisa-cel }\end{array}$ & $\begin{array}{l}\text { CR: } 28 / 61 \\
\text { PR: } 9 / 61\end{array}$ & Lugano & $\begin{array}{l}\text { CRS: } 5 / 61 \\
\text { ICANS: } 6 / 61\end{array}$ & ASTCT/ASTCT & 42 \\
\hline Holtzman & 2020 & 45 & $60(26-75)$ & 35 DLBCL, 3 PMBCL, 7 tFL & axi-cel & $\begin{array}{l}\text { CR: 22/45 } \\
\text { PR: NP }\end{array}$ & NP & $\begin{array}{l}\text { CRS: NP } \\
\text { ICANS: } 18 / 45\end{array}$ & NP/CTCAE 4.03 & 43 \\
\hline Strati & 2020 & 100 & $60(18-85)$ & LBCL (Including 77 DLBCL) & axi-cel & $\begin{array}{l}\text { CR: NP } \\
\text { PR: NP }\end{array}$ & Lugano & $\begin{array}{l}\text { CRS: } 9 / 100 \\
\text { ICANS: } 41 / 100\end{array}$ & CARTOX/CARTOX & 44 \\
\hline Faramand & 2020 & 75 & $63(23-79$ & $\begin{array}{l}50 \text { DLBCL, } 25 \text { Transformed } \\
\text { Indolent lymphomas }\end{array}$ & axi-cel & $\begin{array}{l}\text { CR: 36/68 } \\
\text { PR: 29/68 }\end{array}$ & Lugano & $\begin{array}{l}\text { CRS: } 12 / 75 \\
\text { ICANS: } 23 / 75\end{array}$ & $\begin{array}{l}\text { ASTCT/CARTOX, } \\
\text { ASTCT, CTCAE v4.03 }\end{array}$ & 45 \\
\hline
\end{tabular}


TABLE 2 | Continued

\begin{tabular}{|c|c|c|c|c|c|c|c|c|c|c|}
\hline $\begin{array}{l}\text { First } \\
\text { Author }\end{array}$ & Year & No. & $\begin{array}{l}\text { Median age } \\
\text { (range)-yr }\end{array}$ & Histological type & CAR-T type & $\begin{array}{c}\text { Efficacy } \\
\text { evaluation }\end{array}$ & Scale & $\begin{array}{l}\text { Toxicity evaluation } \\
\text { (grade } \geq 3 \text { ) }\end{array}$ & Scale (CRS/ICANS) & Ref \\
\hline Kittai & 2020 & 9 & $64(40-77)$ & $\mathrm{RS}$ & axi-cel & $\begin{array}{l}\text { CR: } 8 / 8 \\
\text { PR: } 5 / 8\end{array}$ & Lugano & $\begin{array}{l}\text { CRS: } 1 / 9 \\
\text { ICANS: } 3 / 9\end{array}$ & ASTCT/ASTCT & 46 \\
\hline Deng & 2020 & 24 & $58(24-74)$ & 16 DLBCL, 6 tFL, 2 PMBCL & axi-cel & $\begin{array}{l}\text { CR: NP } \\
\text { PR: NP }\end{array}$ & NP & $\begin{array}{l}\text { CRS: } 4 / 24 \\
\text { ICANS: } 12 / 24\end{array}$ & NP/NP & 47 \\
\hline Dean & 2020 & 96 & $64(19-79)$ & $\begin{array}{l}47 \text { DLBCL, } 15 \text { HGBCL, } 5 \\
\text { PMBCL, } 29 \text { NP }\end{array}$ & axi-cel & $\begin{array}{l}\text { CR: } 74 / 96 \\
\text { PR: } 63 / 96\end{array}$ & NP & $\begin{array}{l}\text { CRS: } 9 / 96 \\
\text { ICANS: } 28 / 96\end{array}$ & Lee/CTCAE 4.03 & 48 \\
\hline Sermer & 2020 & 69 & $63(19-85)$ & DLBCL & $\begin{array}{l}47 \text { axi-cel, } 22 \\
\text { tisa-cel }\end{array}$ & $\begin{array}{l}\text { CR: 50/69 } \\
\text { PR: 36/69 }\end{array}$ & Lugano & $\begin{array}{l}\text { CRS: NP } \\
\text { ICANS: NP }\end{array}$ & NP/NP & 49 \\
\hline Wudhikarn & 2020 & 60 & $63(20-86)$ & DLBCL & $\begin{array}{l}43 \text { axi-cel, } 17 \\
\text { tisa-cel }\end{array}$ & $\begin{array}{l}\text { CR: NP } \\
\text { PR: NP }\end{array}$ & NP & $\begin{array}{l}\text { CRS: } 7 / 60 \\
\text { ICANS: } 13 / 60\end{array}$ & NP/NP & 50 \\
\hline Rubin & 2020 & 204 & $60+-12^{*}$ & Inexact ${ }^{\#}$ & axi-cel & $\begin{array}{l}\text { CR: NP } \\
\text { PR: NP }\end{array}$ & NP & $\begin{array}{l}\text { CRS: NP } \\
\text { ICANS: } 51 / 204\end{array}$ & NP/CTCAE 4.03 & 51 \\
\hline
\end{tabular}

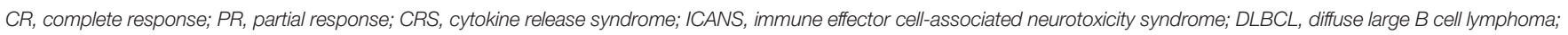

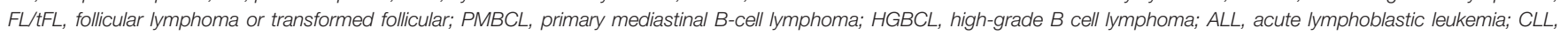

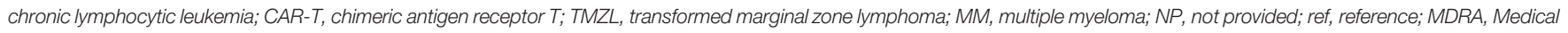

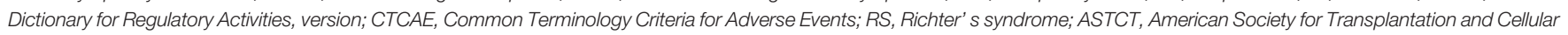

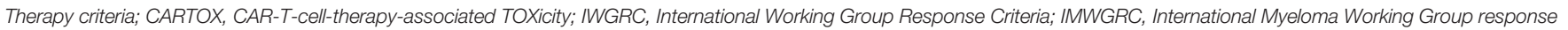
criteria; IWG on CLL RC, International Workshop Group on CLL response criteria.

Independent scale: the institution used their own criteria instead of international criteria, which can be found in original text.

${ }^{\dagger}$ Very good partial response was analyzed as complete response.

"The statement in original text was severe CRS, but it was not clear if it was $\geq$ grade 3 and therefore not included for analysis.

*No separate CR and PR numbers were provided.

* Mean \pm standard deviation.

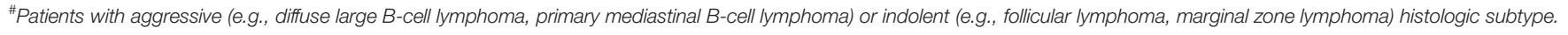

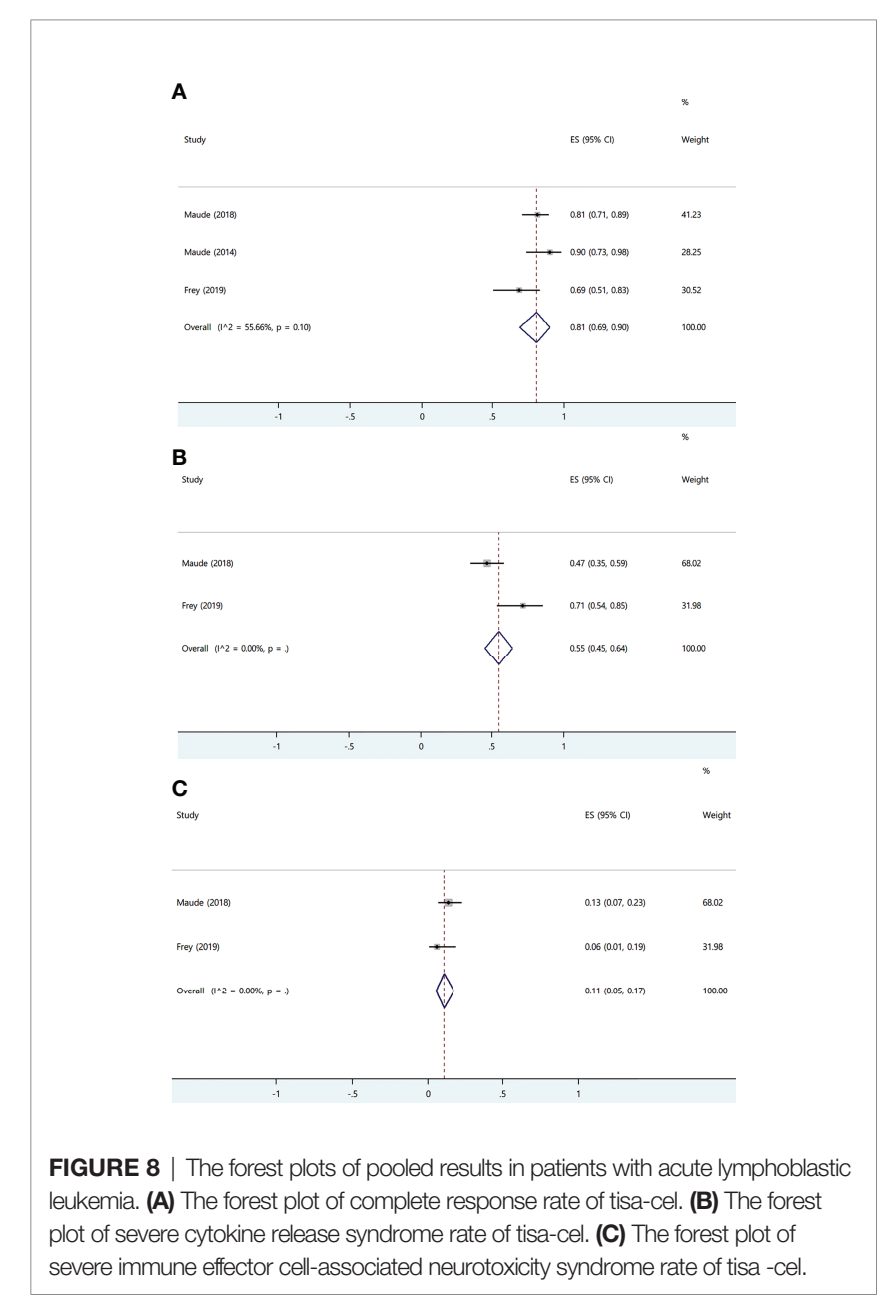

including the meta-analysis and the systematic review section, was run with data from the full-text version of the TRANSCEND NHL 001 trial and is therefore unaffected. In addition, two footnotes were ignored in Table 2. The corrected Table 2 appears below.

In the original article, the neurotoxicity result of tisa-cel in the Primary Mediastinal B Cell Lymphoma subgroup was incorrectly stated. A correction has been made to Results, Primary Mediastinal B Cell Lymphoma, Paragraph 1.

"A study on tisa-cel that included one patient with PMBCL with central nervous system (CNS) involvement indicated that the patient was showing ongoing response at day 90 and developed only grade 1 CRS and no ICANS".

Secondly, a percentage was carelessly written incorrectly in the comparison of severe cytokine release syndrome between adult and pediatric patients with acute lymphoblastic leukemia. A correction has been made to Results, Acute Lymphoblastic Leukemia and Chronic Lymphocytic Leukemia, Paragraph 3.

"Adult patients with ALL were more likely to develop grade $\geq 3$ CRS than pediatric patients (71 vs $47 \%$, respectively)".

In addition, we carelessly wrote "tisa-cel" instead of "liso-cel" and used a wrong percentage in one place. A correction has been made to Discussion, Paragraph 5.

"In the TRANSCEND NHL 001 trial, 7\% of patients received non-conforming products, two patients experienced manufacturing failure of liso-cel, and $10 \%$ of patients died before receiving liso-cel."

In the original article, there was a mistake in the legend for Figure 8 as published. "tisa-cel" was carelessly written as "axicel". The correct legend appears below.

The authors apologize for these errors and state that this does not change the scientific conclusions of the article in any way. The original article has been updated. 


\section{REFERENCES}

1. Abramson JS, Palomba ML, Gordon LI, Lunning MA, Wang ML, Arnason JE, et al. Pivotal Safety and Efficacy Results From Transcend NHL 001, A Multicenter Phase 1 Study of Lisocabtagene Maraleucel (Liso-Cel) in Relapsed/Refractory (R/R) Large B Cell Lymphomas. Blood (2019) 134 (Supplement_1):241. doi: 10.1182/blood-2019-127508

2. Abramson JS, Palomba ML, Gordon LI, Lunning MA, Wang M, Arnason J, et al. Lisocabtagene Maraleucel for Patients With Relapsed or Refractory Large B-Cell Lymphomas (TRANSCEND NHL 001): A Multicentre Seamless Design Study. Lancet (2020) 396:839-52. doi: 10.1016/S0140-6736(20) 31366-0
Publisher's Note: All claims expressed in this article are solely those of the authors and do not necessarily represent those of their affiliated organizations, or those of the publisher, the editors and the reviewers. Any product that may be evaluated in this article, or claim that may be made by its manufacturer, is not guaranteed or endorsed by the publisher.

Copyright (c) 2021 Meng, Wu, Sun, Xun, Liu, Hu and Huang. This is an open-access article distributed under the terms of the Creative Commons Attribution License (CC BY). The use, distribution or reproduction in other forums is permitted, provided the original author(s) and the copyright owner(s) are credited and that the original publication in this journal is cited, in accordance with accepted academic practice. No use, distribution or reproduction is permitted which does not comply with these terms. 